\title{
$\begin{array}{ll}\text { Research Square } & \text { Preprints are preliminary reports that have not undergone peer review. }\end{array}$ or referenced by the media as validated information. \\ Predicting the probability of avian reproductive success and its components at a nesting site
}

\author{
Sinchan Ghosh \\ Indian Statistical Institute
}

Arnab Banerjee

Indian Statistical Institute

\section{Soumalya Mukhopadhyay}

Visva-Bharati University: Visva-Bharati

\section{Santanu Ray}

Visva-Bharati University: Visva-Bharati

Sabyasachi Bhattacharya ( $\nabla$ sabyabhatta@gmail.com )

Indian Statistical Institute https://orcid.org/0000-0002-6432-6988

\section{Research Article}

Keywords: Merops philippinus, Conservation strategy, Breeding behavior, Principal component analysis, Deep learning, Socioecology

Posted Date: February 22nd, 2022

DOI: https://doi.org/10.21203/rs.3.rs-1313546/v1

License: (c) (i) This work is licensed under a Creative Commons Attribution 4.0 International License.

Read Full License 


\title{
Predicting the probability of avian reproductive success and its components at a nesting site
}

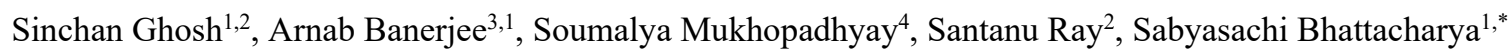 \\ ${ }^{1}$ Agricultural and Ecological Research Unit, Indian Statistical Institute, Kolkata, India
}

${ }^{2}$ Systems Ecology and Ecological Modelling Laboratory, Department of Zoology, Visva-Bharati, Santiniketan, Birbhum, India

${ }^{3}$ Department of Mathematics, Jadavpur University, Kolkata, India

${ }^{4}$ Department of Statistics, Visva-Bharati, Santiniketan, Birbhum, India

${ }^{*}$ Corresponding author, email: sabyabhatta@gmail.com

\begin{abstract}
Avian reproduction has three chronological components: nesting, mating, hatching, and fledging. Predicting the probability of individual components helps to identify the period of reproduction that needs the most aid, increasing the conservation efficiency. This prediction requires identification of biotic, abiotic, and sociological variables of a bird's environment responsible for these componentwise success probabilities. There is also no standard methodology to estimate these probability values separately. This study estimates the absolute success probability of each component, identifies correlated environmental predictors and gives a modeling framework to accurately predict the success probabilities using Merops Philippines as a test bed. The result using surveyed data and proposed methodology indicates the corridor between nesting and mating is most vulnerable to the environment. Social structure is the key to all reproductive components but nesting. Both biotic and abiotic factors are crucial determinants of nesting success. Mating, hatching, and fledging success depend more on biotic factors than abiotic ones. Linear modeling frameworks are helpful to explore which types of environment are a better determinant of the success of a reproductive component. Artificial neural networking is more useful to predict the successes of a new site. Although developed using Merops philippinus data, the proposed methodology and modeling framework are also applicable for other birds.
\end{abstract}

\section{Key words}

Merops philippinus; Conservation strategy; Breeding behavior; Principal component analysis; Deep learning; Socioecology

\section{Significance statement:}

This study proposes a new methodology to estimate individual success at different phases of avian reproduction. This study provides a clear pathway to identify social and environmental factors responsible for these successful reproduction events and accurately predict the successes using the identified criteria. This study illustrates the overall procedure using Merops philippinus, a long-distance migratory cooperative bird as a testbed.

\section{Statements and Declarations}

Data and code availability: The primary data and codes used in this study are available as supplementary material. Labels of the figures have been edited using MS office PowerPoint 2019.

Funding: Author S. Ghosh received financial support from the Council of Scientific and Industrial Research, Government of India (file no. 09/093(0184) 2019-EMR-I). Author Arnab Banerjee acknowledges the DSKPD Fellowship of University grants commission (no. BL/17-18/0490) and SERB NPDF postdoctoral fellowship (File No. - PDF/2021/000604).

Competing interests: The authors declare no competing interests.

Ethical approval: Merops philippinus is the least concerned species as per the International Union for Conservation of Nature and the reproductive data collection process was purely observational. Soil sample collected from the field did not contain nests of any birds within it. No animals were harmed or disturbed during data collection. Data have been collected as per The Wild Life (Protection) Amendment Bill, Ministry: Environment, Forests and Climate Change, Government of India.

Informed consent: This study is a part of the research project sanctioned by the Council of Scientific and Industrial Research, India ( file no. 09/093(0184) 2019-EMR-I) and evaluated by the Three Members Assessment Committee (DS/20-21/0448), Indian Statistical Institute. 


\section{Introduction}

1 Sexual reproduction not only grows and sustains the population, but also increases the variation in traits of the population (Birkhead, Fletcher, \& Pellatt, 1998; Peterson, Angert, \& Kay, 2020). Therefore, reproductive success is critical to the growth, maintenance, and evolution of sexually breeding species and is helpful for conservation policymakers (Peterson, Angert, \& Kay, 2020). There are three main strategies for the sexual reproduction of animals: Oviparity, Viviparity, and Ovo-viviparity. Oviparous animals lay eggs, which hatch outside their body and may require incubation by the animals. Ovoviviparous incubates eggs inside the body and gives birth to offspring hatched from the eggs. Viviparous animals develop offspring from the eggs and give birth to offspring capable of free-living (Blackbum, 1999). The body's internal environment of animals is more stable than the external environment, especially for endothermic animals (Kvarnemo \& Forsgren, 2000; Canals, 1998). So the reproductive success of the oviparous animals, such as birds, are more prone to environmental fluctuations such as seasonal changes than the viviparous animals (Andreasson, Nilsson, \& Nord, 2020; Nilsson $\&$ Nord, 2021). Thus predicting and estimating the reproductive success of viviparous animals such as birds requires considering seasonal variability corresponding to each reproductive behavior.

Locating a nesting site is the first and foremost requirement of a bird at the start of the breeding season (Fontaine \& Martin, 2006; Maia, Brasileiro, Lacava, \& Macedo, 2012). Upon arriving at the nesting site, most birds start to build nests and find their potential partners (Hansell, 2000; Von Haartman, 1957). To find their potential partners, birds show nuptial behaviors such as dancing, singing, food-offering, and nest building (Hansell, 2000; Murphy, 2007). Many birds, being a sex-biased population, have extra males who cannot find females for mating (Kokko, Johnstone, \& TH, 2001; Koenig \& Dickinson, 2004). These inferior males settle down as helpers and receive food, shelter, and sometimes the opportunity to breed with an already paired female as the repayment of their cooperation (Kokko, Johnstone, \& TH, 2001; Reyer, 1986). The helpers cooperate with the breeding birds by protecting their eggs from predators, parasites, and other environmental hazards (Stacey, Koenig, \& others, 1990; Koenig \& Dickinson, 2004). The joint effort of breeders and helpers leads most of the eggs to successfully hatch (Reid, Monaghan, \& Ruxton, 2000; Mumme, 1992). The newly hatched chicks (hatchlings) are still prone to environmental risks (Heg \& van der Velde, 2001; Wiebe \& Bortolotti, 1995; Jones \& Ward, 2021; Wiebe \& Martin, 2000). So breeder and helper birds continue to raise the hatchlings till these chicks can fly (Heg \& van der Velde, 2001). Finally emerging from the nests as successful flying young birds (fledgling), the chicks at the end of the breeding season are the fittest in their generation (Koenig \& Dickinson, 2004; Stacey, Koenig, \& others, 1990). Thus the reproductive success of a bird has four components: Nesting, mating, hatching, and fledging.

Overall, these four components depend on the environment of the nesting site and the social structure of the bird population (Heg \& van der Velde, 2001; Stacey, Koenig, \& others, 1990; Ghosh, Al Basir, Chowdhury, Bhattacharya, \& Ray, 2021; Rebar, Barbosa, \& Greenfield, 2019). These four components show different degrees of dependence on the determining factors (Hall, 2004; Malueg, Walters, \& Moore, 2009; Arroyo, 2002). Any information on the decline in the success of a specific component of a site may help conservation biologists to prevent the loss in reproductive success and subsequent risk of species extinction. Since monitoring reproductive success by surveying the entire breeding zone is costly and labor-intensive, predicting the overall reproductive success and its components based on environmental and social determinants is an easy alternative for the extinction risk assessment (Higginson, 2017; Lewis, 1981; Ghosh, Al Basir, Chowdhury, Bhattacharya, \& Ray, 2021). Such a prediction is possible through a modeling framework and some prior knowledge. This prior knowledge involves information about the environment, avian society, and estimates of componentwise reproductive success. Surprisingly the existing literature has no standard methodology to estimate the absolutely componentwise reproduction success as this prior knowledge. So before proceeding to modelling, the establishment of a methodology to estimate the componentwise nesting success is necessary.

Mathematical or dynamic models can predict reproductive success over time for a particular site (Aresu, Pennino, De Rosa, Rotta, \& Berlinguer, 2021; Ghosh, Al Basir, Chowdhury, Bhattacharya, \& Ray, 2021), and statistical models such as regression and machine learning can predict the success for the various site at a particular time using environmental and social factors (Gokcekus, et al., 2021; Elith, Hastie, Dudik, Chee, \& Yates, 2011).

Note 
that different environmental and social factors, the determinants of reproductive success, vary over different nesting sites (Rebar, Barbosa, \& Greenfield, 2019; Stacey, Koenig, \& others, 1990; Aresu, Pennino, De Rosa, Rotta, \& Berlinguer, 2021; Aviles, Stokke, Moksnes, Roskaft, \& Moller, 2007). Rates of change of these determinants with time may also change radically at different sites (Carey, 2009; Michener, Blood, Bildstein, Brinson, \& Gardner, 1997). Due to ecological succession, multiple new environmental factors may arise or disappear at these nesting sites over time (Michener, Blood, Bildstein, Brinson, \& Gardner, 1997). Also, two subsequent years may experience two different social structures in migratory birds. Such a random environment may introduce too much uncertainty in the long-term predictions. So dynamic modeling may not be an excellent choice to study the reproductive success of birds considering multiple environmental factors. However, prediction of nesting success is possible for a shorter period due to less chance of random environmental change, such as a particular season over different sites. Therefore, the statistical models can perform well to predict reproductive success and assess the quality of a nesting site using environmental predictors. Regression models are better predictors with few determining environmental factors and extensive survey data. Machine learning overcomes the lacunae of data volume, but hardly explains the underlying relationship between environmental factors, social structure, and components of reproductive success (Singh, Kaur, \& Malhotra, 2009).

Although the literature on avian reproduction, its society, and related environmental condition is vast, most key determinants and their relationships with each of the components of reproductive success are still under the veil. For example, long-distance summer migratory bird Merops philippinus (blue-tailed bee-eater) is known to rely on soil characteristics for nest selection (Wang, et al., 2009; DeCandido, 2010). However, the factors associated with mating, hatching, and fledging are yet unknown. Since these birds burrow their nests in the soil, their nests are known to be insulated and may eliminate the effect of weather on these reproductive phases. Still, there may be the prey, predators, or other factors that may influence these reproductive phases. Also, two or more environmental factors may share a linear correlation between themselves. For example, soil properties and vegetation, determinants of the nesting sites, often correlate between themselves ( $\mathrm{Li}$, Wang, Zhang, \& Zhang, 2021). In such a case, any one of the correlated determinants may predict the success to the same or different degree, but using all of them will not provide any further improvement prediction. Identifying such relationships is necessary before modeling reproductive success with environmental factors (Elith, Hastie, Dudik, Chee, \& Yates, 2011).

Considering the lacunae in the existing literature, this study focuses on two objectives: (a) Establish a standard method to estimate the componentwise reproductive success. (b) Identify abiotic, biotic, and social variables for nesting sites that may influence components of reproductive success. (c) Provide a framework to predict the probability of reproductive success and its components using the identified environmental and social variables.

\section{Methodology}

This section first discusses the data collection procedure for abiotic and biotic factors. Then we propose a new standardized methodology to estimate the componentwise reproductive success within this section to meet the first objective. The data collection of social structure is incorporated within this method. Finally, we describe the statistical procedure to identify the significant predictors followed by the modelling framework for the prediction used in this study.

This study uses Merops philippinus (blue-tailed bee-eater, henceforth BTBE) as a testbed. To collect data on the nesting of BTBE, we survey the entire Bhagirathi-Hooghly delta and riverine belts in the northern part of West Bengal, India from 2018 to 2019. We identify six major nesting sites with colonies larger than 30 individuals. We selected five brooding colonies with no interactions in between them. Additionally, we chose two more sites where the birds arrived but did not reproduce. One of these two sites appeared with the attempts of nesting but without nests.

\section{Abiotic data collection}

We collected soil samples from all the nesting sites and brought them back to the laboratory in labeled and sealed polyethylene bags. These samples were later sieved through a $2 \mathrm{~mm}$ sieve to prepare a soil-water suspension of a $1: 2.5$ weight ratio. We measured $\mathrm{pH}$, inorganic carbon, organic carbon and nitrogen content, cation exchange capacity, calcium, magnesium, potassium, sodium, iron, and total base saturation for all the samples. We determined sand, silt, and clay percentages by the International Pipette Method (Olmstead, Alexander, \& Middleton, 1930). We estimated the amount of organic carbon (C) through Nelson \& Sommers 

nitrogen, cation exchange capacity (CEC), Calcium, Sodium, Potassium, and Magnesium (Begheijn, 1980).

We extracted the Iron and Aluminium content with dithionite-citrate-bicarbonate (DCB) solution for free sesquioxides and measured them using an atomic absorption spectrometer. In addition to our surveyed data, we measured the same advice variables for other successful nesting sites from the available literature. We measured the vegetation density and average height per nest of colonies in the nesting sites. We counted the number of herbs, shrubs, and trees per square meter in the nesting area. Finally, we counted anthropogenic artifacts (e.g., Magnetic tower) surrounding surveyed nesting sites.

\section{Biotic data collection:}

We collected 300 bird pellets of regurgitated materials from seven different observation sites. We dissected the pellets and identified the insect exoskeletons to confirm the prey taxa observed during their foraging behavior. Since these birds are superior in competition and competitors and predators are rarely found in the surveyed field, the collection of biotic factors focuses mainly on the prey of these birds.

For example, we found some snakes killed by group defense of BTBE, trying to prey on them at one site. Since we found no broken eggshells, signs of dead chicks, or snake-crawlings in the nests, considering such predators or any other interactive species may make predictions erroneous. We discarded any data of such interactive species if they appeared at only one site and had less than 5\% samples/observation points considering those species as accidental.

Methodology to estimate the component-wise reproductive success:

We observationally counted the number of birds and the number of nests they built for the breeding season. Since per nest, two breeders reside, the number of adult helpers is number of adult birds at site $-2 \times$ number of nests with eggs or chicks. We observationally counted the number of eggs and chicks in the breeding nests throughout the breeding season. Since reproductive success has four components, we determine the probability of each component in the following steps.

We counted the number of complete, incomplete, and abandoned nests per site to determine the nest-site selection success. If a site has $C_{N}$ complete nests, $I_{N}$ incomplete nests, and $A_{N}$ abandoned nests, the nesting success of a site in terms of probability is

$$
P(N)=\frac{C_{N}+A_{N}}{C_{N}+A_{N}+I_{N}} .
$$

We count the number of eggs per nest after breeding to determine the mating success of the birds. If $M$ is the number of nests with mating pair, is the observed egg count, and $E_{M}$ is the expected egg count per mating pair nests, given nesting has already occurred, the mating success in terms of probability at the site is-

$$
P(M \mid N)=\frac{1}{t} \sum_{i=2}^{t} \frac{O_{M}(t)}{E_{M}(t)},
$$

A BTBE breeding pair can lay up to six eggs upon mating. Therefore per breeder, the expected maximum egg count is three. The female bird may lay eggs asynchronously throughout the season. Considering the continuous mating and asynchronous egg-laying, we calculated the expected egg count as follows-

$$
\left.E_{M}(t)=(B(t)-B(t-1)) \times 3+B(t-1) \times 3-o_{\text {total }}(t)\right)
$$

where,

$$
o_{\text {total }}(t)=\left(O_{M}(t-1)+O_{H}(t-1)+O_{F}(t-1)\right)+o_{M}-\left(O_{H}(t)-O_{H}(t-1)\right)+\left(O_{F}(t)-O_{F}(t-1)\right)
$$

Here, $B(t)$ is the breeder count per nest during the observation at $t$ time point; $B(t-1)$ is the breeder count per nest during the observation at the previous time point $t-1$. $O_{\text {total }}$ is the total number of offspring at the $t$ time point. We count the newly hatched chicks per site to determine the hatching success rate. If a nest has $O_{H}$ hatchlings and the expected to have $E_{H}$ hatchlings, the hatching success in terms of probability at the site is 


$$
P(H \mid M, N)=\frac{1}{t} \sum_{i=2}^{t} \frac{O_{H}(t)}{E_{H}(t)},
$$

Hatching is asynchronous due to asynchronous mating. The newly hatched birds leave the nest after learning to fly. So we calculate the expected hatchlings as follows-

$$
E_{H}=O_{M}(t-1)+O_{H}(t-1)-\left(O_{F}(t)-O_{F}(t-1)\right) .
$$

Here, the $O_{F}$ is the observed flying chicks from a nest at a time point. Flying chicks are the fledgling of the breeding pair. We expected to find all hatchlings, from the previous time point to fledge ( $E_{F}$ is the expected fledgling) at the time of observation. We calculate the fledgling success as follows-

$$
P(F \mid H, M, N)=\frac{1}{t} \sum_{i=2}^{t} \frac{O_{F}(t)}{E_{F}(t)},
$$

where $E_{F}=O_{F}(t-1)+O_{H}(t-1)$.

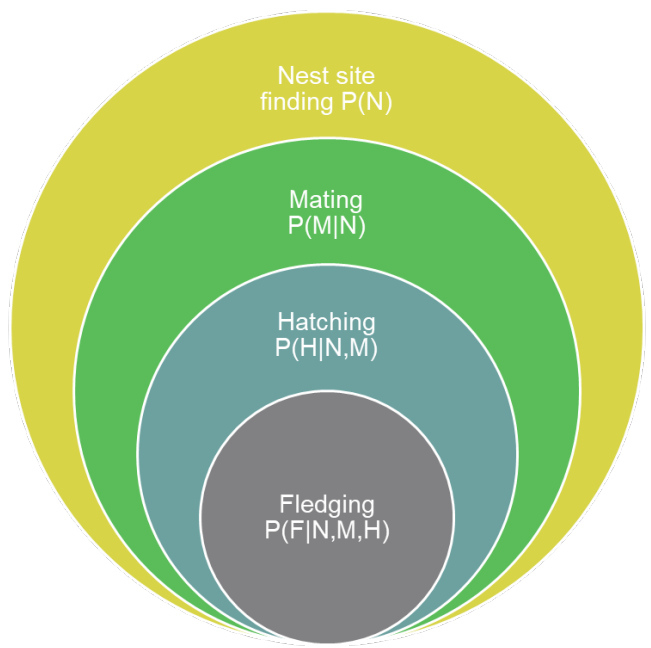

Figure 1: Venn diagram showing the relationships among components of reproductive success

Note that these estimated probabilities of reproductive components except nesting, depend on the probabilities of its previous components (Figure 1). The overall reproductive success found in the field is the fledging success dependent upon its previous events. Unless we estimate the independent probabilities of each component, the true determinant of the success of a particular reproductive component is impossible. Therefore, we estimate independent probabilities of all the components as follows-

Since a component of reproductive success can not take place at a site where the previous component has no occurrence, we can say that $P(H \mid M)=P(H \mid N, M), \mathrm{P}\left(\mathrm{M} \cap N^{c}\right)=0, \mathrm{P}\left(\mathrm{H} \cap M^{c}\right)=0, \mathrm{P}\left(\mathrm{F} \cap H^{c}\right)=0$. As per the probability chain rule, we can the independent probability of fledgling success as $P(F)=P\left(H^{c}\right) P\left(F \mid H^{c}\right)+P(H) P(F \mid H)=P(M) P(H \mid M) P(F \mid H)$, the independent probability of hatching success as $P(H)=P\left(M^{c}\right) P\left(H \mid M^{c}\right)+P(M) P(H \mid M)$, the independent probability of the mating success as $P(M)=P(N) P(M \mid N)$. We calculate all the independent probabilities of success for each reproductive component using these estimates and the field data. We use the hierarchical clustering method based on the complete euclidian distance between means of the environmental variables to profile the sites (Hartigan, 1975). If the proposed method can estimate componentwise reproductive successes correctly, nesting sites with similar environments to support similar components of reproductive success should be grouped together.

Statistical procedure and modelling framework:

We use the pairwise Pearson's correlation test to check the correlation between identified environmental factors from the field and the components of reproduction success. Pearsons correlation finds linear correlations; multiple linearly correlated variables introduce errors within a single linear model if used as predictors. One way to reduce the number of correlated variables for prediction without information loss is Principal Component 
Analysis (PCA). So, we synthesize principal components from environmental variables using Q-mode-PCA to reduce the variables as alternative uncorrelated predictors to reduces errors during modelling (biplots of PCA in

178 Appendix A).

179 We use a series of linear regression and artificial neural networks with and without linear output (ANN-L and 180 ANN-NL) to predict different components of reproductive success. We build each regression models using all 181 different sets of predictors. Since neural networks performs better with more information, we use all predictors 182 identified in the field, all predictors linearly correlated with the successes, and all correlated predictors of either biotic or abiotic category. We evaluate and compare the predictions of these models using the root mean square error (RMSE).

Since the number of hidden layers and the number of nodes in a hidden layer can have a range of values. This topology of the network is important for the prediction of ANNs. We search for the topology for the best predictions by searching the least RMSE inside a grid: number of nodes in the first hidden layer $\times$ number of nodes in second hidden layer following the algorithm in Stathakis (2009). For each topographic structure of the ANNs, we iterate each of the models 500 times with various ratios of training-test data. All the ANNs use backpropagation learning method with each repeated iterations (finalized model structures are in Appendix B).

192 All models have been built in R software using "neuralnet" package (Fritsch, 2019).

\section{Result:}

194 Estimates of componentwise nesting success:

195 Figure 2 shows the 8 sites found with the birds' presence from the field survey, classified based on the profile of their environmental data. The overall reproductive success and componentwise reproductive successes listed under each site show the estimated probability to observe successful reproduction decreases over each phase of reproduction. The position of Site A, B, C, and $\mathrm{H}$ cannot be justified by only overall reproductive success in this classification. However, the estimated absolute probabilities of componentwise reproductive success through the proposed method show the environment of sites $\mathrm{A}$ and $\mathrm{H}$ did not support reproductions after nesting. The success probabilities also drastically decrease for sites B and C. The nesting success of site $\mathrm{H}$ is much higher than the other three sites. Site F had many incomplete, but no complete and abandoned nests. As a result, the estimated nesting success is zero. So the environment of sites $\mathrm{H}$ and $\mathrm{F}$ must support the immigration of birds at first but eventually turns out to be adverse for mating and consequent events. The correlated environmental factors in these sites can indicate how the loss in success occurs. 
Classification of sites based environmental variables

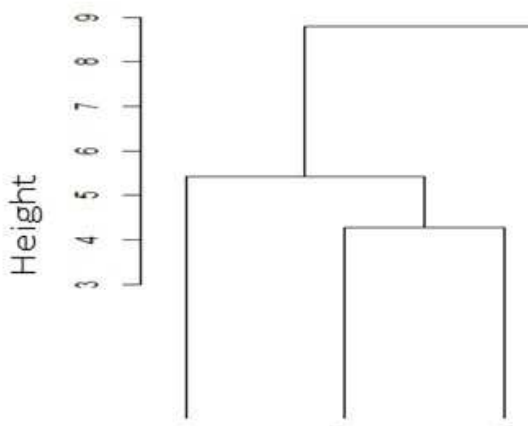

Site id: $\mathbf{E}$

D

Reproductive 0.5728976

success

Nesting $\quad 0.87301587$

Success

Mating

success

Hatching

success

Fledging

success

0.27394855

0.10898796

G

0.39267576

0.28509136

0.21934679

$0.21934679 \quad 0.15873461$

206

Figure 2: Componentwise estimated successes and classification of identified sites from field survey based on the profiling of environmental factors.

\section{Identified predictors:}

Figure 3 shows all the identified predictors of reproductive success and its components from the field survey and their pairwise correlation. Only significantly correlated variables can predict reproductive success or its components through linear models. Identified variables with insignificant correlations can still predict the nesting success through networking as they might share a nonlinear relationship with the successes. 


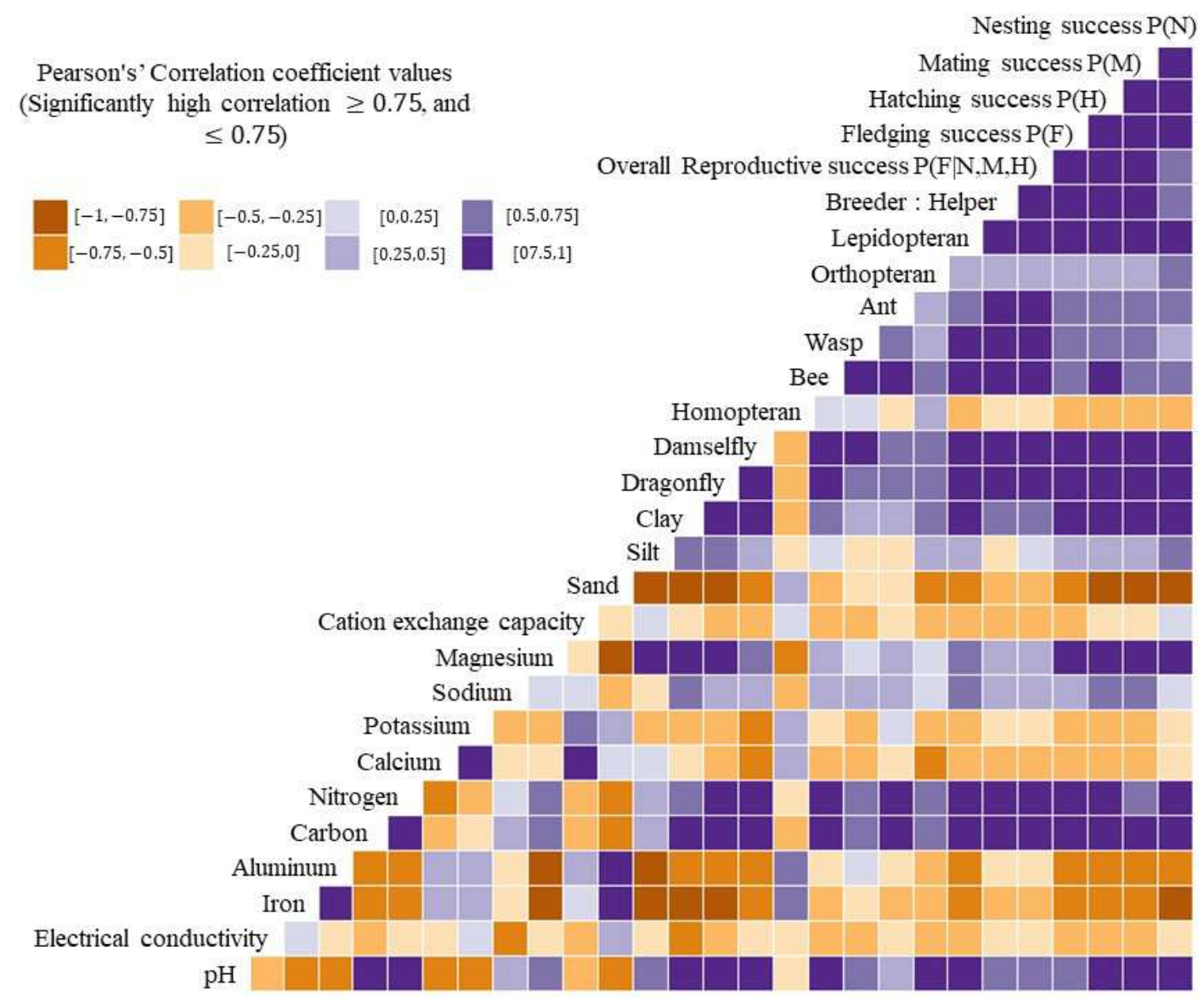

Figure 3: Correlogram of identified predictors of reproductive success and its components.

The social factor, the breeder and helper ratio at breeding sites, in particular, is the key predictor of all components of reproductive success. The linear correlation between this ratio and nesting success is 0.8 . Otherwise, all components of reproductive success are fully correlated with this ratio. However, this ratio is highly correlated with most biotic predictors.

Among all the biotic factors, prey species are the key predictors of all the components of reproductive success. Eight groups of prey have been identified from pellets: Dragonfly, Damselfly, Lepidopterans, Bees, Wasps, and Black ants. Six groups are highly associated with overall reproductive success. Nesting, mating, and fledging successes are significantly associated with only three biotic predictors for linear regression: Lepidopteran, Damselfly, and Dragonflies. Similarly, Lepidopteran, Bee, Dragonfly, and Damselfly are predictors of hatching success in linear regression. However, some of these prey species coexist in all nesting sites and thus are correlated to each other. So principal components have been synthesized from each set as predictors. The components with the highest maximum variance are used as the predictor of linear regression. Also, these biotic variables may again depend upon identified abiotic predictors as some of them are also correlated.

Topographic variables are the key biotic predictors of reproductive success and its components of this bird. Carbon and Nitrogen content of soil are two major predictors of overall reproductive success. The significantly correlated topographic factors to predict nesting are the clay, sand, magnesium, carbon, nitrogen, iron, and $\mathrm{pH}$. For mating success, these predictors are the same excluding nitrogen and iron. For hatching success, the predictors are the same as nesting success except for iron. The fledging success predictors are the same as hatching success excluding sand for linear models. Since these factors have multicollinearity, the principal components synthesized from them are used as the final predictor in linear regressions.

There are four sets of principal components to predict overall reproductive, nesting, mating, hatching and fledging successes. Each set of principal components can predict one success. Each set consists of three subsets: one synthesized from all predictors, one synthesized from bots only, one synthesized from abiotic variables only (See Appendix A). 
Comparisons of prediction accuracies:

Figure 4 shows the RMSE of predictions through linear regressions using Principal Components (PC) synthesized from various sets of correlated predictors in terms of RMSE values. Overall reproductive success prediction is most accurate for the PC composed of all correlated variables. Nesting success prediction is most accurate for PC composed of all biotic variables. PCs synthesized from all correlated variables and only correlated biotic variables predict the mating success with the least RMSE, and therefore high accuracy. The result indicates a prediction of hatching success is best using PCs from either all correlated predictors or correlated biotic predictors. Similarly, fledging success is predicted with comparatively low accuracy with only PC from correlated abiotic variables. Since consideration of correlated biotic variables only can give an accurate prediction in these two cases, a conservation biologist can rely on only prey abundance data to predict these two 250 successes.

\section{RMSE values of linear regressions with principal components}

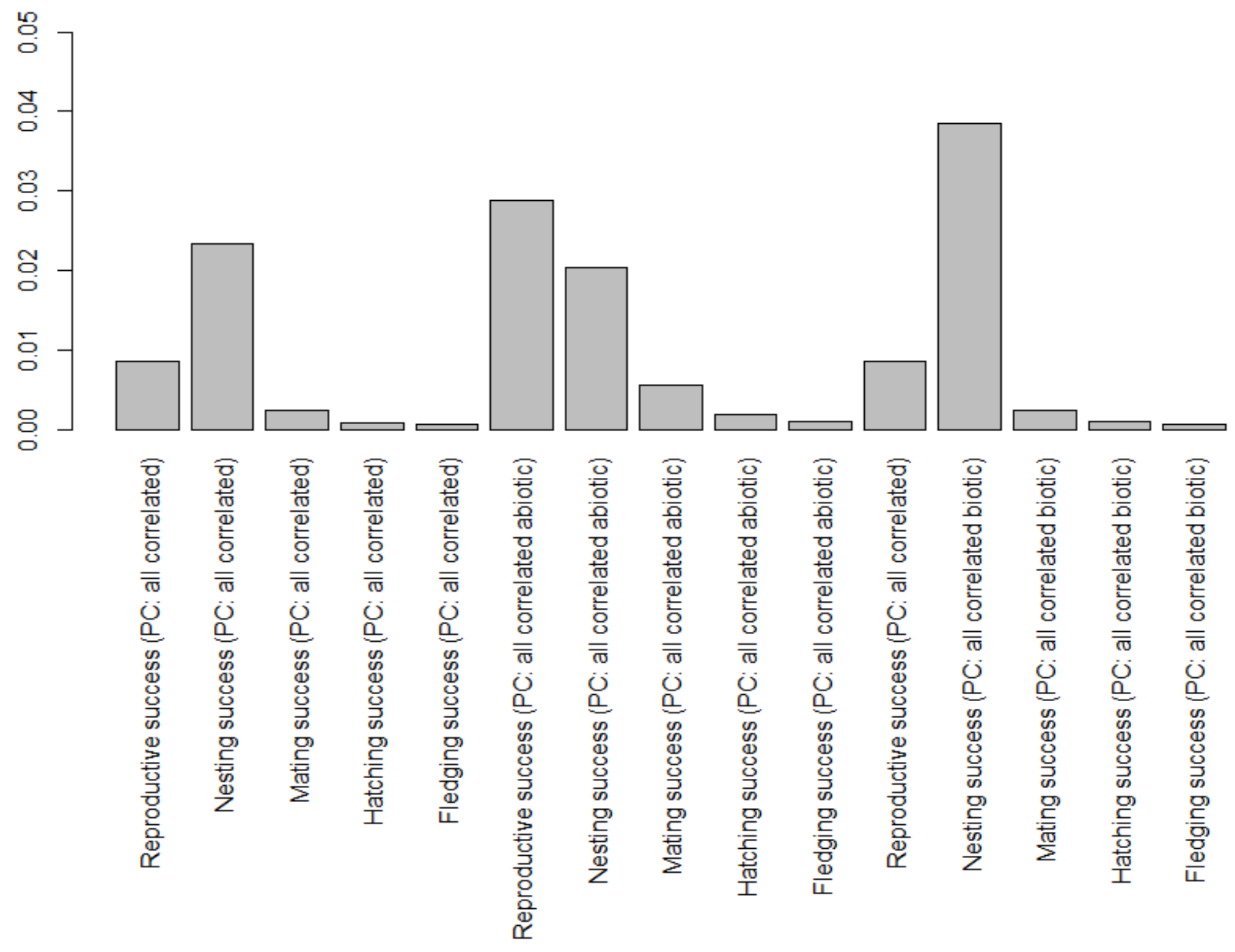

Figure 5 shows the model preference for accurate predictions of reproductive success and its components if some predictors are suspected to share a nonlinear correlation with the successes. The overall reproductive success may share such a correlation as it is most accurately predicted through ANN-L with all identified predictors. However, the nesting success is prediction is still accurate for correlated predictors only. The mating success may also have some nonlinearly correlated predictors as it has higher accuracy in the prediction considering all identified environmental and social factors in the field. Prediction of hatching success is best through the ANN-L uses the correlated predictors. Fledging success prediction is comparatively the same for all neural networks. Since the linear regression models have far less RMSE than all the ANNs to predict hatching and fledging successes, the ANN is a poor choice to predict these two successes only. However, the RMSE of ANNs can be further reduced by repeated training. Therefore, these ANNs are a better choice for predicting components of reproductive success over further training. 


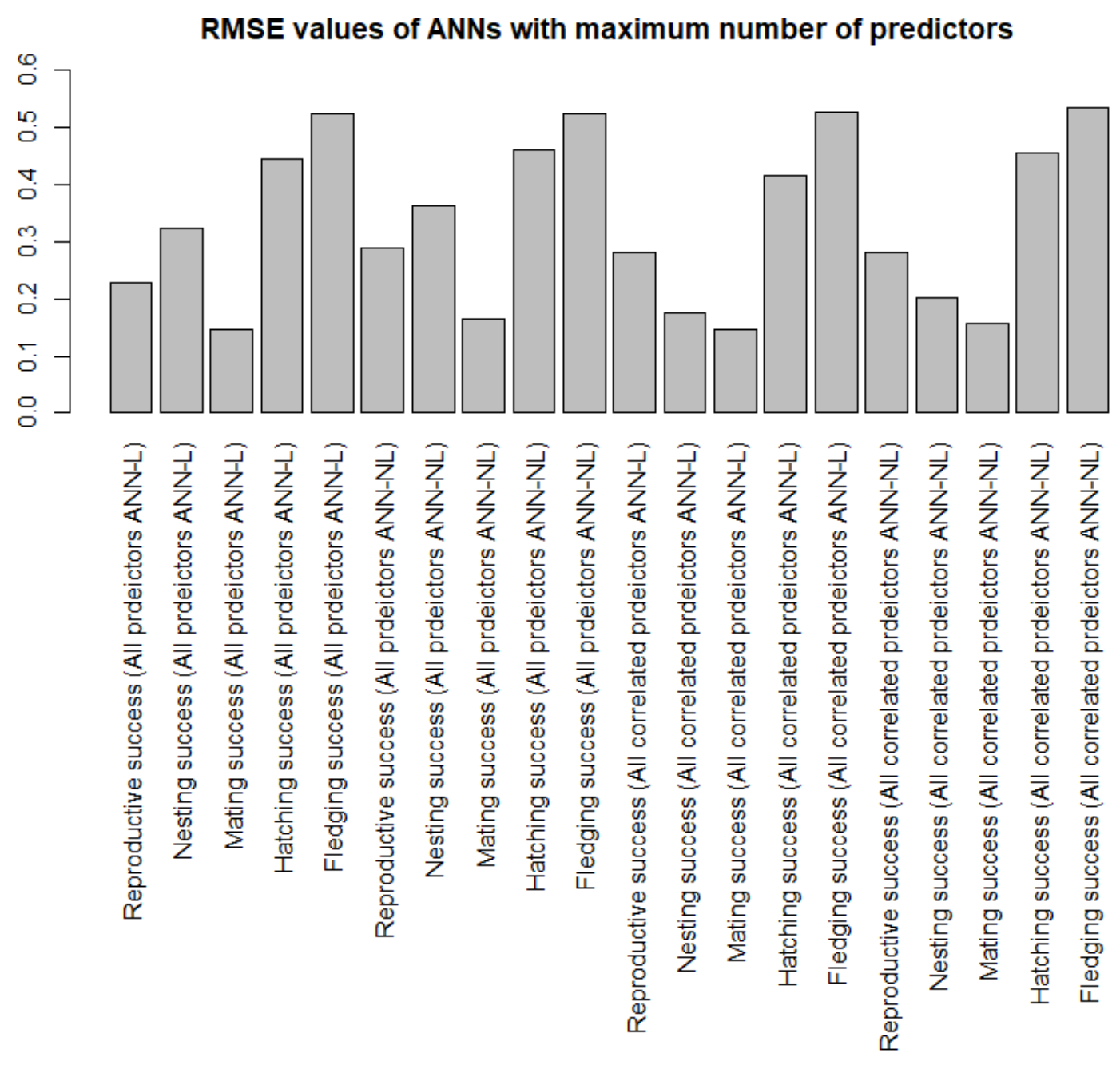

Figure 5: RMSE of ANNs for predicting Reproductive success and its components

\section{Discussion and conclusion}

268 Identification of nesting sites with a high probability of successful reproduction is a key to develop conservation strategy for migratory birds. Predicting the probability of overall reproductive success may help to identify the crucial nesting sites, but the precision of the conservation period requires the knowledge of the probability of each component of reproduction (nesting, mating, hatching, and fledging). Estimating componentwise success is not substantial in the existing literature. So we have developed a measure to estimate the component-wise nesting success. Hierarchical clustering of the observed sites reveals that the corridor between nesting and mating is the most crucial period of reproduction for our testbed species Merops philippinus. Sites $\mathrm{F}$ and $\mathrm{H}$ experienced an adverse environment during the nesting. Sites A, C, and B experienced adverse environments after nesting. One explanation for such environmental adversity may be associated with the social structure of immigrating birds. Ghosh, Al Basir, Chowdhury, Bhattacharya, \& Ray (2021) showed that too many immigrating helpers can decrease prey abundance than immigrating breeders. So these sites might have experienced so high helper immigration that the biotic resource became scarce for breeders to mate and continue reproduction in those sites.

We have identified the environmental and social predictors of componentwise reproductive success using data from a field survey. The result suggests nesting success does not significantly associate with social structure, but other components of reproductive success do. Note that Ghosh, Al Basir, Chowdhury, Bhattacharya, \& Ray (2021) used a nonlinear dynamic model to observe the association between social dynamics and reproduction. However, identification of such underlying nonlinear function is not always possible. 
Lepidopterans, damselfly, and dragonfly are the key prey to sustain all stages of reproduction. The hatching success of this bird is highly associated with hatching success only. During hatching, the birds tend to stay in nests and incubate the eggs more than foraging for prey. Bees are abundant near nests on flowering plants. So, this association may be the result of a higher abundance of bees during this season rather than prey preference. Previous studies already observed an association between soil and nest site selections. Our study finds the association between other components of reproductive success and soil properties. Among all the other soil properties, the high association of carbon and nitrogen content with components of reproduction may have a two-way relationship with this bird's reproduction components. The birds may come to the nesting site following high carbon, nitrogen components, then they increase the contents through fecal matter.

The soil properties associated with reproductive components after nesting may work either by determining the supporting associated biotic factors or by determining the stability of the nests over time. Since linear models can predict the mating, hatching, and fledging works better using biotic predictors, they are more responsible for these latter successes supporting the previous statement. The accuracies of linear models indicate that only nesting success indeed may depend upon both biotic and abiotic predictors. Although linear regression models have much less RMSE than ANNs, they may not perform well for new sites as the value of these principal components synthesized from the dataset will change for a new set of predictors. Also, they fail to capture unknown nonlinear relationships between any predictors and the reproduction. On the other hand selection of a previously trained ANN model is a safe choice for this purpose. Conservation biologists can choose the best ANN model using based on our results to predict the probability of success for a new site. The nonlinearly related environmental factors truly determine these successes, especially the overall reproductive success as per our finding through the ANNs. ANN-Ls are a better choice for overall reproductive, nesting, mating, and hatching successes but ANN-NLs are better choices for fledging success predictions.

Although this study uses Merops philippinus as a test bed, all these successes can be estimated for any other bird species also. First, conservation biologists have to estimate these successes following our proposed methodology and survey data for that. Then they have to identify correlated factors and train ANN models. Then the model with the least RMSE can predict the component-wise success of reproduction. If they aim to find out direct causes of incline and decline in successes, then the regression model based on correlated predictors and their principal components is a better choice.

\section{Data and code availability}

The primary data and codes are available in the supplementary material.

\section{Compliance with Ethical Standards}

Funding:

Author S. Ghosh received financial support from the Council of Scientific and Industrial Research, Government of India (file no. 09/093(0184) 2019-EMR-I). Author Arnab Banerjee acknowledges the DSKPD Fellowship of University grants commission (no. BL/17-18/0490) and SERB NPDF postdoctoral fellowship (File No. - PDF/2021/000604).

Conflict of Interests:

The authors declare no competing interests.

Ethical approval

Merops philippinus is a least concerned species as per International Union for Conservation of Nature and the reproductive data collection process was purely observational. The soil sample collected from the field did not contain nests of any birds within it. No animals were harmed or disturbed during data collection. Data have been collected as per The Wild Life (Protection) Amendment Bill, Ministry: Environment, Forests and Climate Change, Government of India.

Informed consent

This study is a part of the research project sanctioned by the Council of Scientific and Industrial Research, India ( file no. 09/093(0184) 2019-EMR-I) and evaluated by the Three Members Assessment Committee (DS/2021/0448), Indian Statistical Institute. 


\section{References}

Andreasson, F., Nilsson, J.-A., \& Nord, A. (2020). Avian reproduction in a warming world. Frontiers in Ecology and Evolution, 8, 337\}.

Aresu, M., Pennino, M. G., De Rosa, D., Rotta, A., \& Berlinguer, F. (2021). Modelling the effect of environmental variables on the reproductive success of Griffon Vulture (Gyps fulvus) in Sardinia, Italy. Ibis.

Arroyo, B. E. (2002). Fledgling sex ratio variation and future reproduction probability in Montagu's harrier, Circus pygargus. Behavioral Ecology and Sociobiology, 52(2), 109-116.

Aviles, J. M., Stokke, B. G., Moksnes, A., Roskaft, E., \& Moller, A. P. (2007). Environmental conditions influence egg color of reed warblers Acrocephalus scirpaceus and their parasite, the common cuckoo Cuculus canorus. Behavioral Ecology and Sociobiology, 61(3), 475-485.

Begheijn, L. (1980). Methods of chemical analyses for soils and waters. Methods of chemical analyses for soils and waters, 3.

Birkhead, T., Fletcher, F., \& Pellatt, E. (1998). Sexual selection in the zebra finch Taeniopygia guttata: condition, sex traits and immune capacity. Behavioral Ecology and Sociobiology, 44(3), 179-191.

Blackbum, D. G. (1999). Viviparity and oviparity: evolution and reproductive strategies. In Encyclopedia of Reproduction (Volume 4) (pp. 994-1003). Academic Press.

Canals, M. (1998). Thermal ecology of small animals. Biological Research, 31, 367-372.

Carey, C. (2009). The impacts of climate change on the annual cycles of birds. Philosophical Transactions of the Royal Society B: Biological Sciences, 364(1534), 3321-3330.

DeCandido, R. a. (2010). Mass northbound migration of Blue-tailed Merops philippinus and Blue-throated M. viridis Bee-eaters in southern Thailand, spring 2007-2008. Forktail, 26, 42-48.

Elith, J. a., Hastie, T., Dudik, M., Chee, Y. E., \& Yates, C. J. (2011). A statistical explanation of MaxEnt for ecologists. Diversity and distributions, 17(1), 43-57.

Fontaine, J., \& Martin, T. (2006). Parent birds assess nest predation risk and adjust their reproductive strategies. Ecology letters, 9(4), 428--434.

Fritsch, S. a. (2019). Package 'neuralnet'. Training of Neural Networks.

Ghosh, S., Al Basir, F., Chowdhury, G., Bhattacharya, S., \& Ray, S. (2021). Is the primary helper always a key group for the dynamics of cooperative birds? A mathematical study on cooperative breeding birds. Ecological Modelling, 459, 109728.

Gokcekus, S., Firth, J. A., Regan, C., Cole, E. F., Lamers, K. P., \& Sheldon, B. C. (2021). Drivers of passive leadership in wild songbirds: species-level differences and spatio-temporally dependent intraspecific effects. Behavioral Ecology and Sociobiology, 75(12), 1-13.

Hall, M. L. (2004). A review of hypotheses for the functions of avian duetting. Behavioral Ecology and Sociobiology, 55(5), 415-430.

Hansell, M. (2000). Bird nests and construction behaviour. Cambridge University Press.

Hartigan, J. A. (1975). Clustering algorithms. (W. a. sons, Ed.) Inc., 113129.

Heg, D., \& van der Velde, M. (2001). Effects of territory quality, food availability and sibling competition on the fledging success of oystercatchers (Haematopus ostralegus). Behavioral Ecology and Sociobiology, $49(2), 157--169$.

Higginson, A. D. (2017). Conflict over non-partitioned resources may explain between-species differences in declines: the anthropogenic competition hypothesis. Behavioral ecology and sociobiology, 71(7), 1-13. 
Jones, T. M., \& Ward, M. P. (2021). Parasitic cowbird development up to fledging and subsequent post-fledging survival reflect life history variation found across host species. Behavioral Ecology and Sociobiology, 75(9), 1-10.

Koenig, W. D., \& Dickinson, J. L. (2004). Ecology and evolution of cooperative breeding in birds. Cambridge University Press.

Kokko, H., Johnstone, R. A., \& TH, C.-B. (2001). The evolution of cooperative breeding through group augmentation. Proceedings of the Royal Society of London. Series B: Biological Sciences, 268(1463), 187--196.

Kvarnemo, C., \& Forsgren, E. (2000). The influence of potential reproductive rate and variation in mate quality on male and female choosiness in the sand goby, Pomatoschistus minutus. Behavioral Ecology and Sociobiology, 48(5), 378-384.

Lewis, D. M. (1981). Determinants of reproductive success of the white-browed sparrow weaver, Plocepasser mahali. Behavioral Ecology and Sociobiology, 9(2), 83-93.

Li, W., Wang, J., Zhang, Y., \& Zhang, M. (2021). A novel characterization on the interaction of soil and vegetation in a reclaimed area of opencast coalmine based on joint multi-fractal method. Ecological Indicators, 121, 107094.

Maia, R., Brasileiro, L., Lacava, R. V., \& Macedo, R. H. (2012). Social environment affects acquisition and color of structural nuptial plumage in a sexually dimorphic tropical passerine. Plos One, e47501.

Malueg, A., Walters, J., \& Moore, I. (2009). Do stress hormones suppress helper reproduction in the cooperatively breeding red-cockaded woodpecker (Picoides borealis)? Behavioral Ecology and Sociobiology, 63(5), 687-698.

Michener, W. K., Blood, E. R., Bildstein, K. L., Brinson, M. M., \& Gardner, L. R. (1997). Climate change, hurricanes and tropical storms, and rising sea level in coastal wetlands. Ecological Applications, 7(3), 770--801.

Mumme, R. L. (1992). Do helpers increase reproductive success? Behavioral Ecology and Sociobiology, 31(5), 319--328.

Murphy, T. G. (2007). Racketed tail of the male and female turquoise-browed motmot: male but not female tail length correlates with pairing success, performance, and reproductive success. Behavioral Ecology and Sociobiology, 61(6), 911--918.

Nelson, D. a., \& Sommers, L. (1983). Methods of soil analysis: Part 2 chemical and microbiological properties. Methods of soil analysis: Part 2 chemical and microbiological properties, 9, 539-579.

Nilsson, J., \& Nord, A. (2021). Variation in reproductive investment increases body temperature amplitude in a temperate passerine. Oecologia, 197(2), 365-371.

Olmstead, L. B., Alexander, L. T., \& Middleton, H. E. (1930). A pipette method of mechanical analysis of soils based on improved dispersion procedure. Technical Bulletin.

Peterson, M. L., Angert, A. L., \& Kay, K. M. (2020). Experimental migration upward in elevation is associated with strong selection on life history traits. Ecology and evolution, 10(2), 612-625.

Rebar, D., Barbosa, F., \& Greenfield, M. D. (2019). Female reproductive plasticity to the social environment and its impact on male reproductive success. Behavioral Ecology and Sociobiology, 73(4), 1-14.

Reid, J. M., Monaghan, P., \& Ruxton, G. (2000). The consequences of clutch size for incubation conditions and hatching success in starlings. Functional Ecology, 14(5), 560--565.

Reyer, H.-U. (1986). Breeder-helper-interactions in the pied kingfisher reflect the costs and benefits of cooperative breeding. Behaviour, 277--303. 
Singh, Y., Kaur, A., \& Malhotra, R. (2009). Comparative analysis of regression and machine learning methods for predicting fault proneness models. International journal of computer applications in technology, 35(2-4), 183-193.

Stacey, P. B., Koenig, W. D., \& others. (1990). Cooperative breeding in birds: long term studies of ecology and behaviour. Cambridge University Press.

Stathakis, D. (2009). How many hidden layers and nodes? International Journal of Remote Sensing, 30(8), 2133--2147.

Von Haartman, L. (1957). Adaptation in hole-nesting birds. Evolution, 339--347.

Wang, Y.-P., Siefferman, L., Wang, Y.-J., Ding, T.-S., Chiou, C.-R., Shieh, B.-S., . . Yuan, H.-W. (2009). Nest site restoration increases the breeding density of blue-tailed bee-eaters. Biological conservation, $142(8), 1748-1753$

Wiebe, K. L., \& Bortolotti, G. R. (1995). Food-dependent benefits of hatching asynchrony in American kestrels Falco sparverius. Behavioral Ecology and Sociobiology, 36(1), 49--57.

Wiebe, K. L., \& Martin, K. (2000). The use of incubation behavior to adjust avian reproductive costs after egg laying. Behavioral Ecology and Sociobiology, 48(6), 463-470. 
$438 \quad$ Appendix A

439 Principal component analysis of biotic factors:

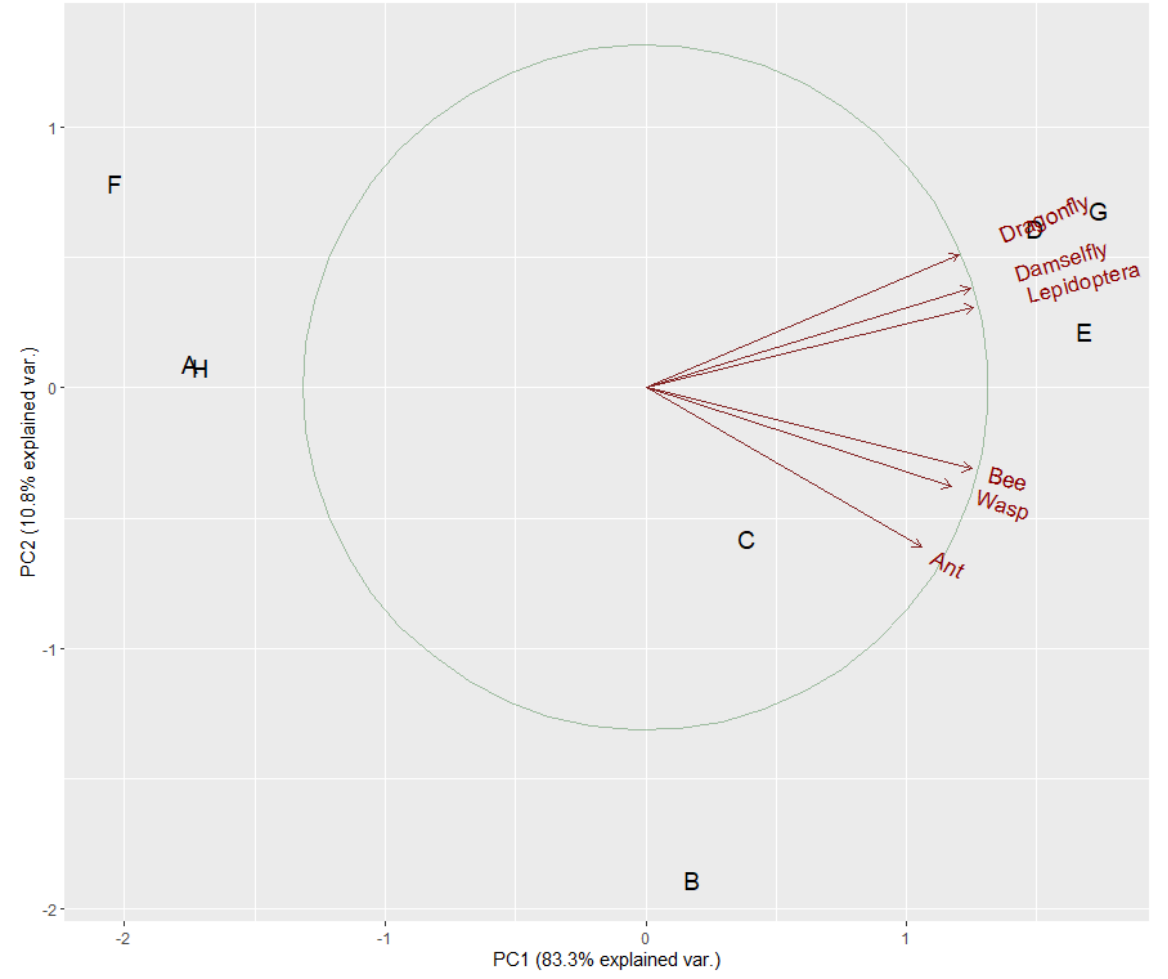

Figure 6: PCA of correlated biotic factors for Reproductive

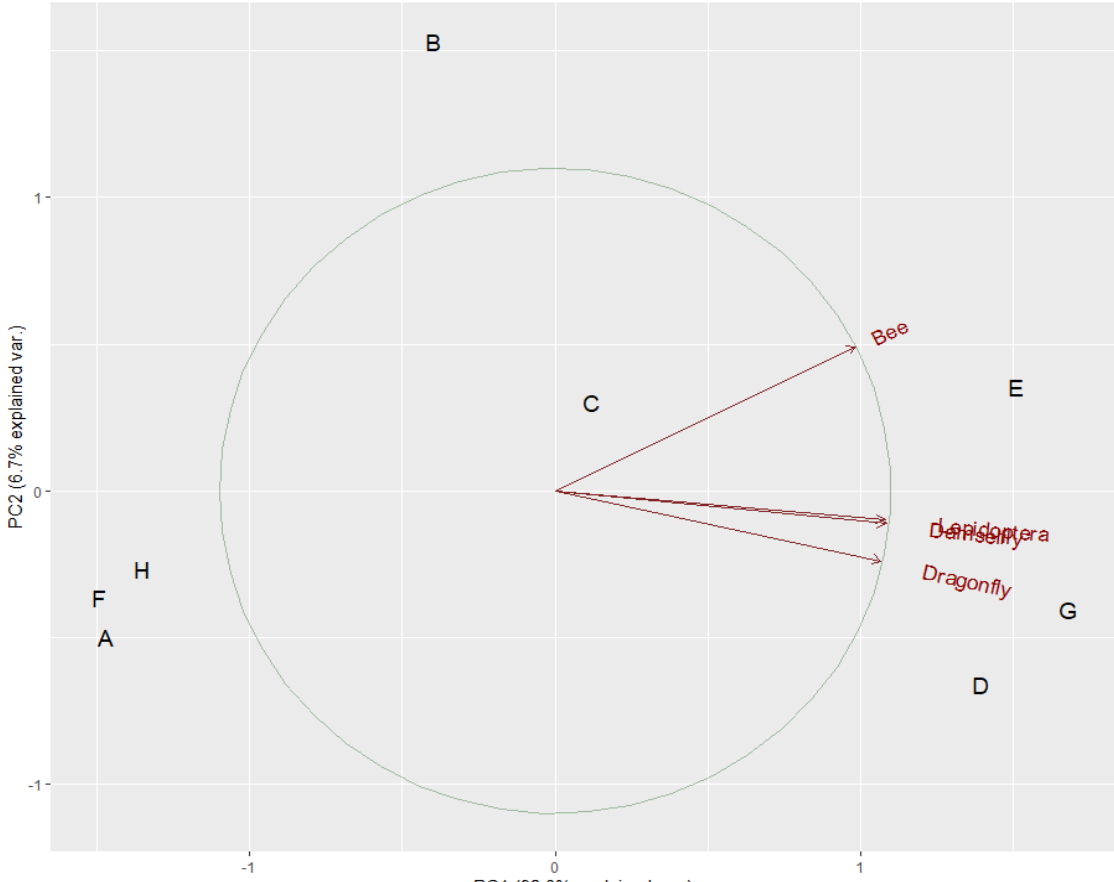




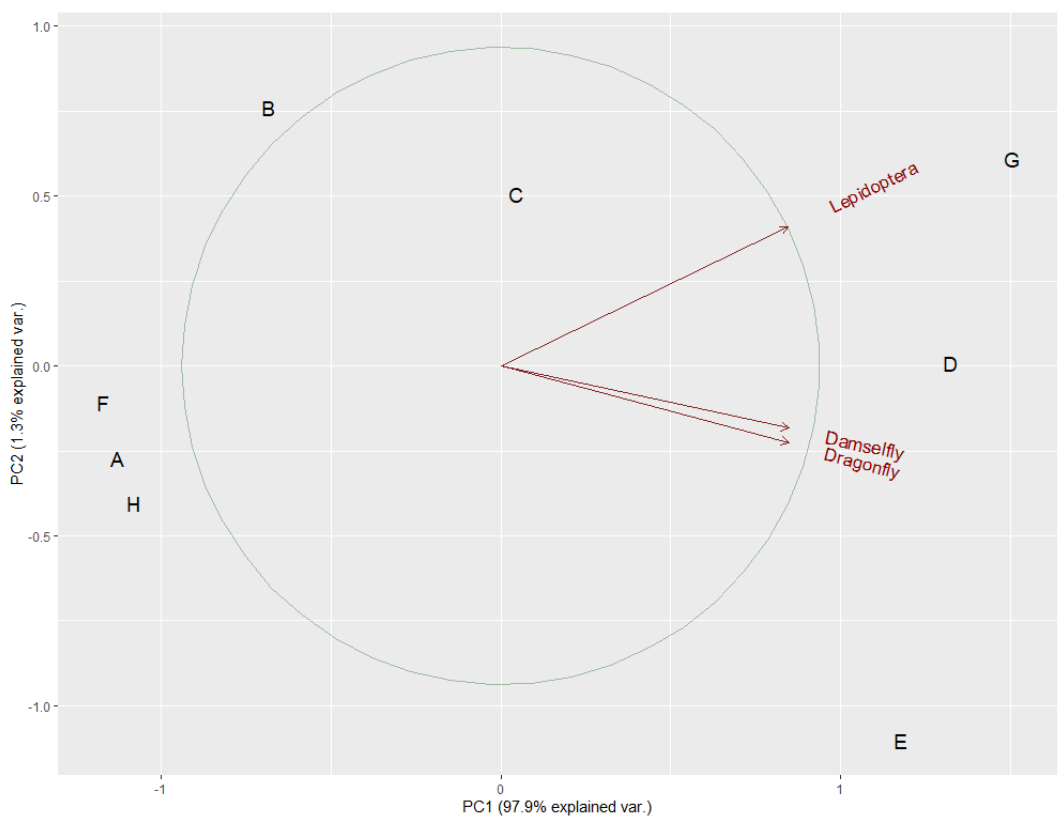

446 Figure 8: PCA of correlated biotic factors for nesting, mating, and fledging successes

447 Principal component analysis of abiotic factors:

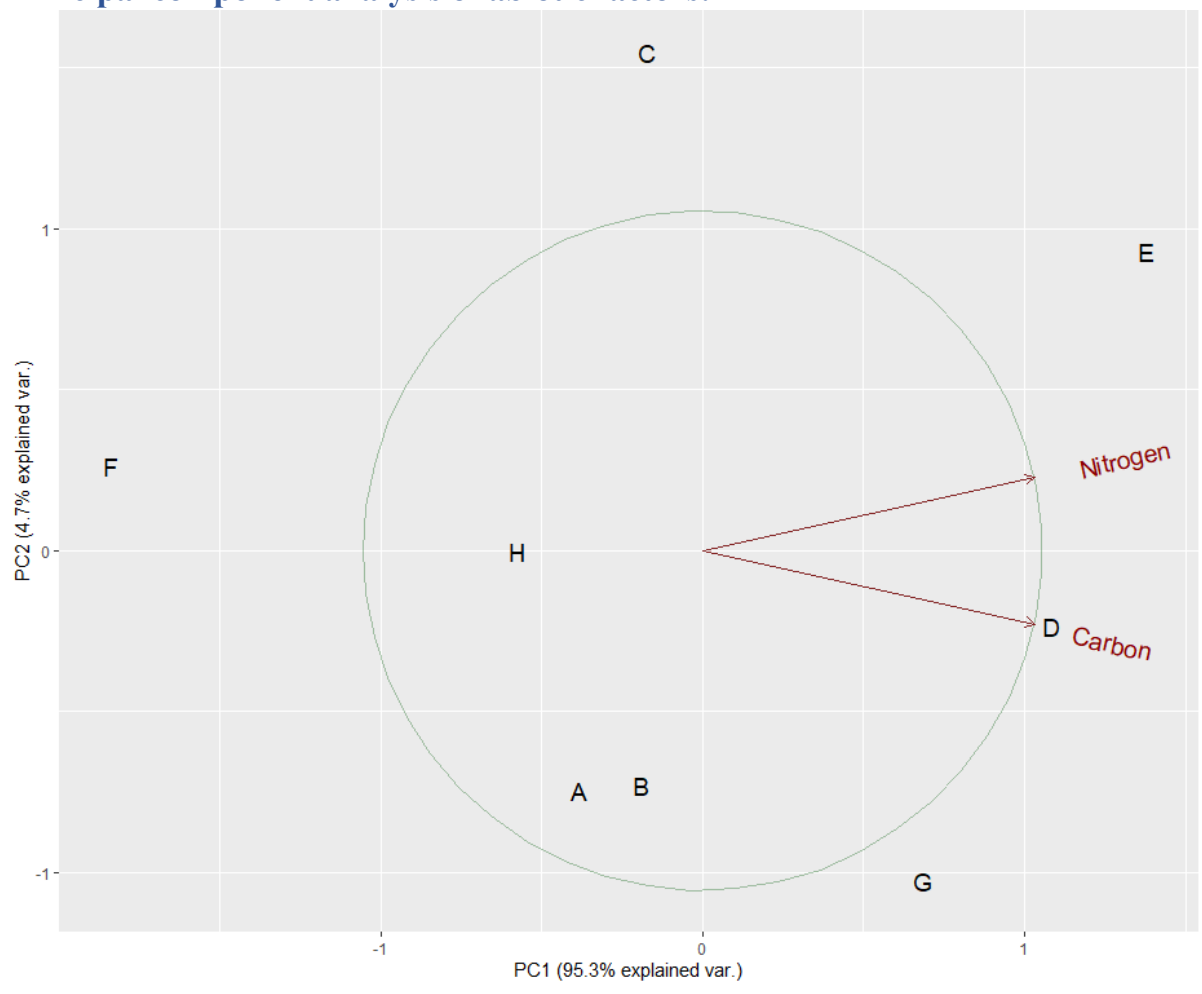




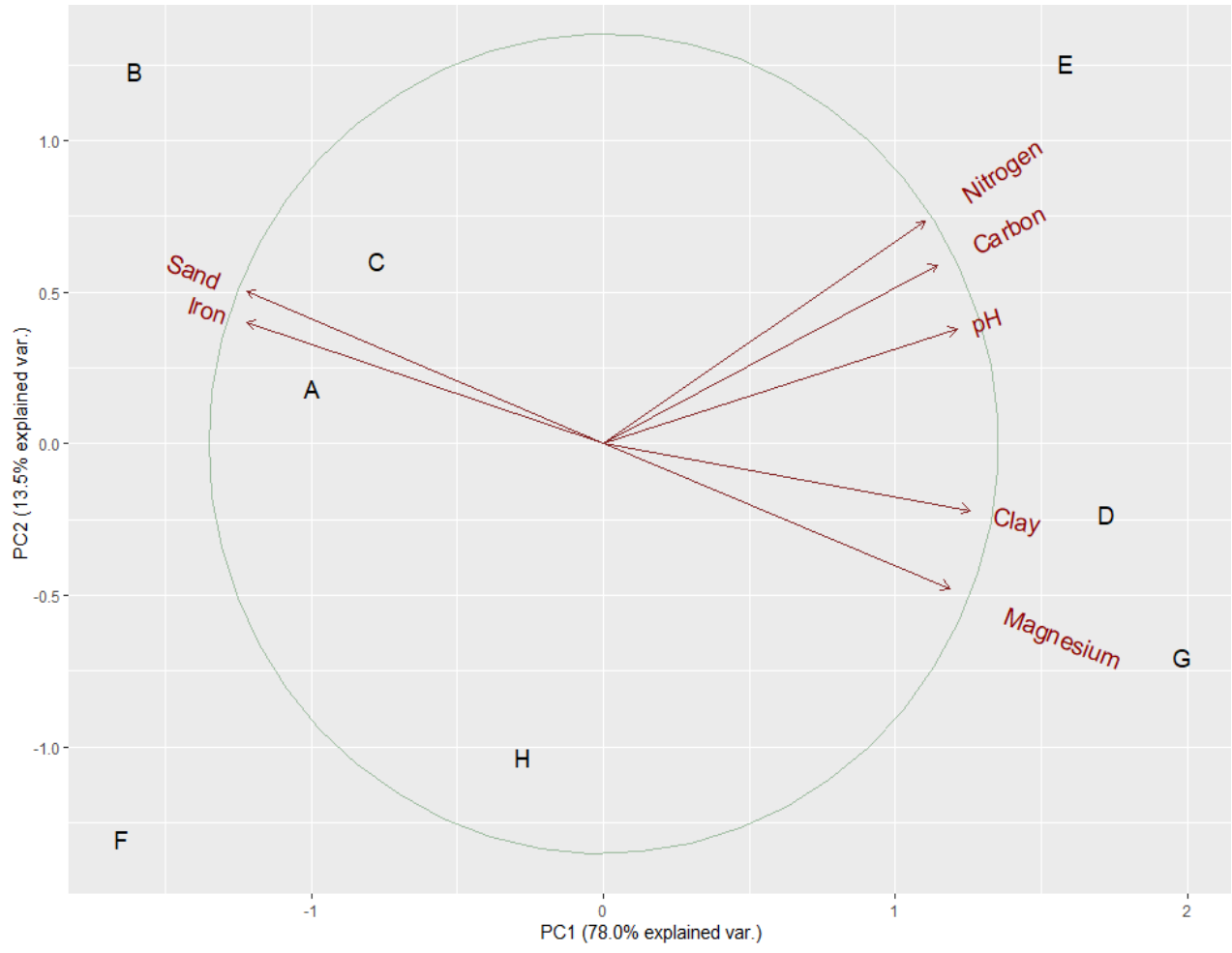

451 Figure 10: PCA of correlated abioticc factors for nesting success

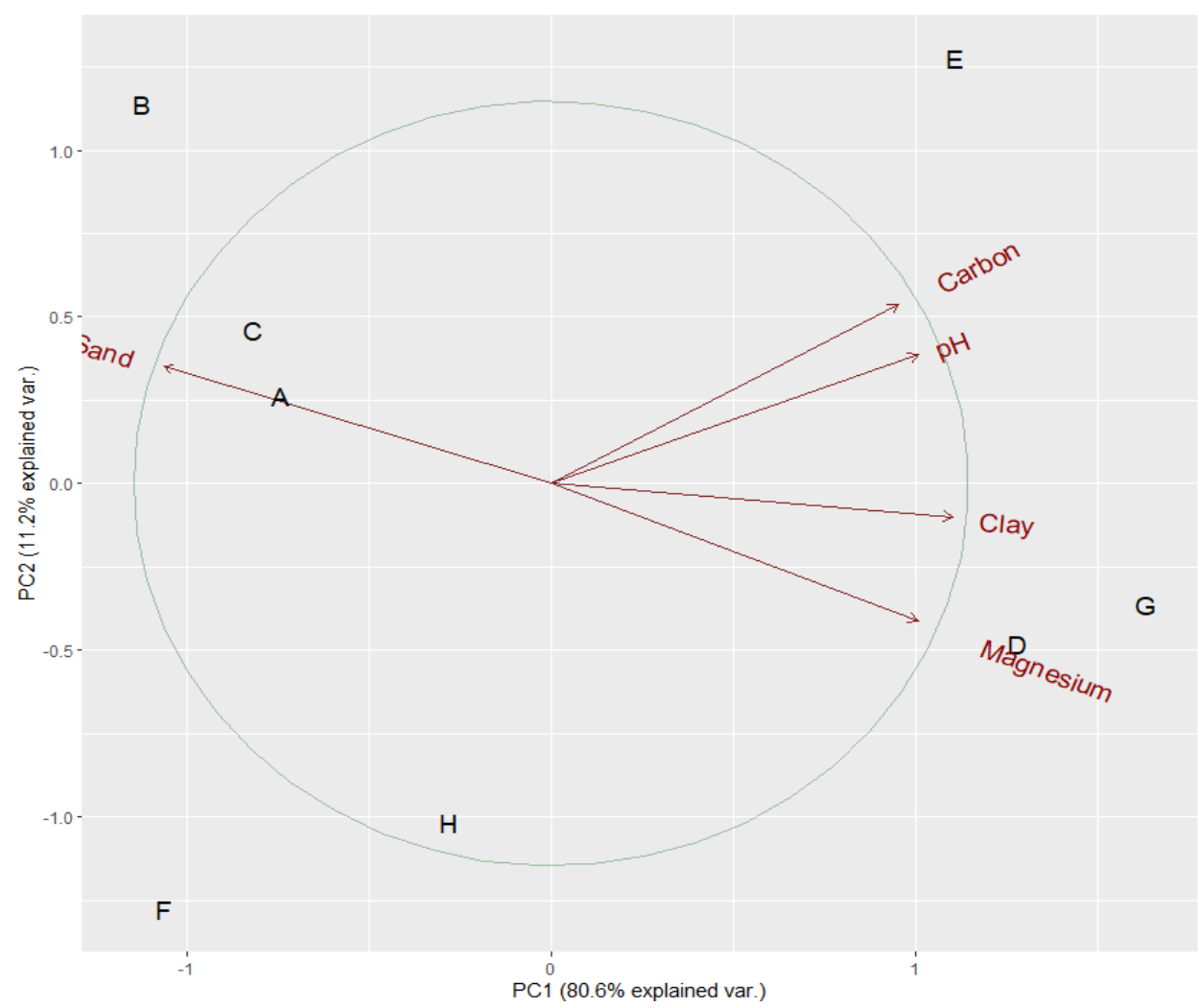




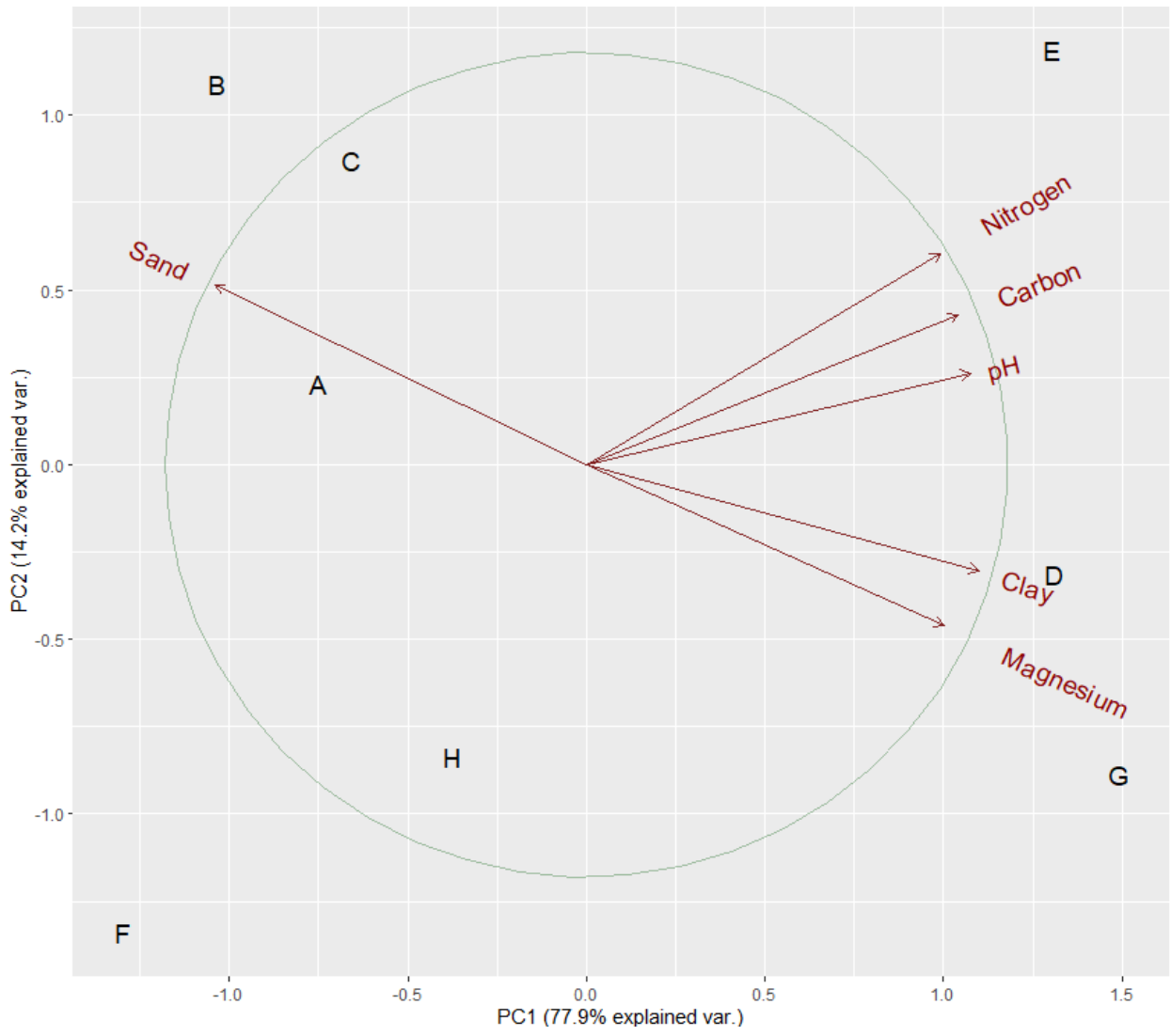

Figure 12: PCA of correlated abiotic factors for hatching success

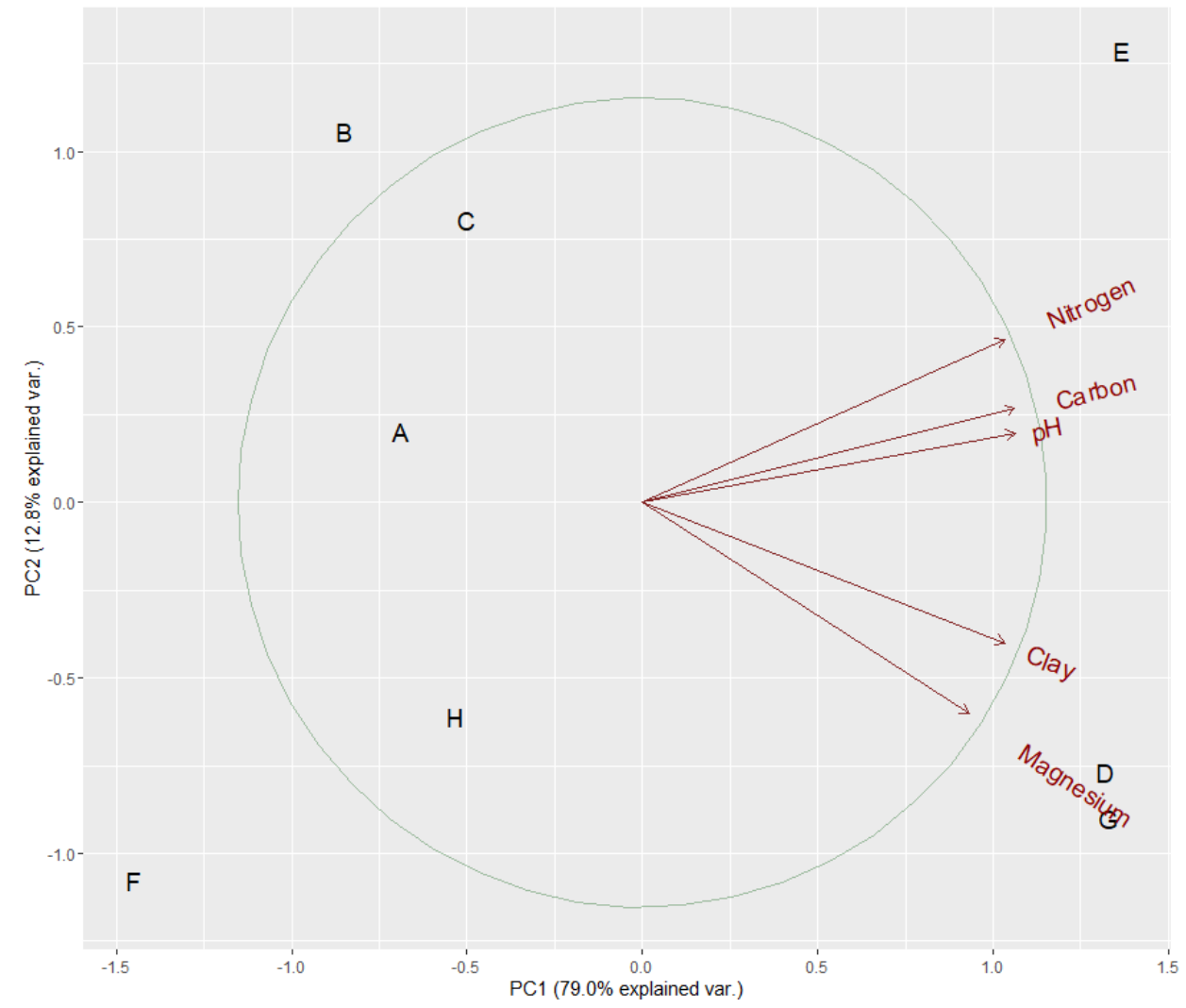

Figure 13: PCA of correlated abiotic factors for fledging success 


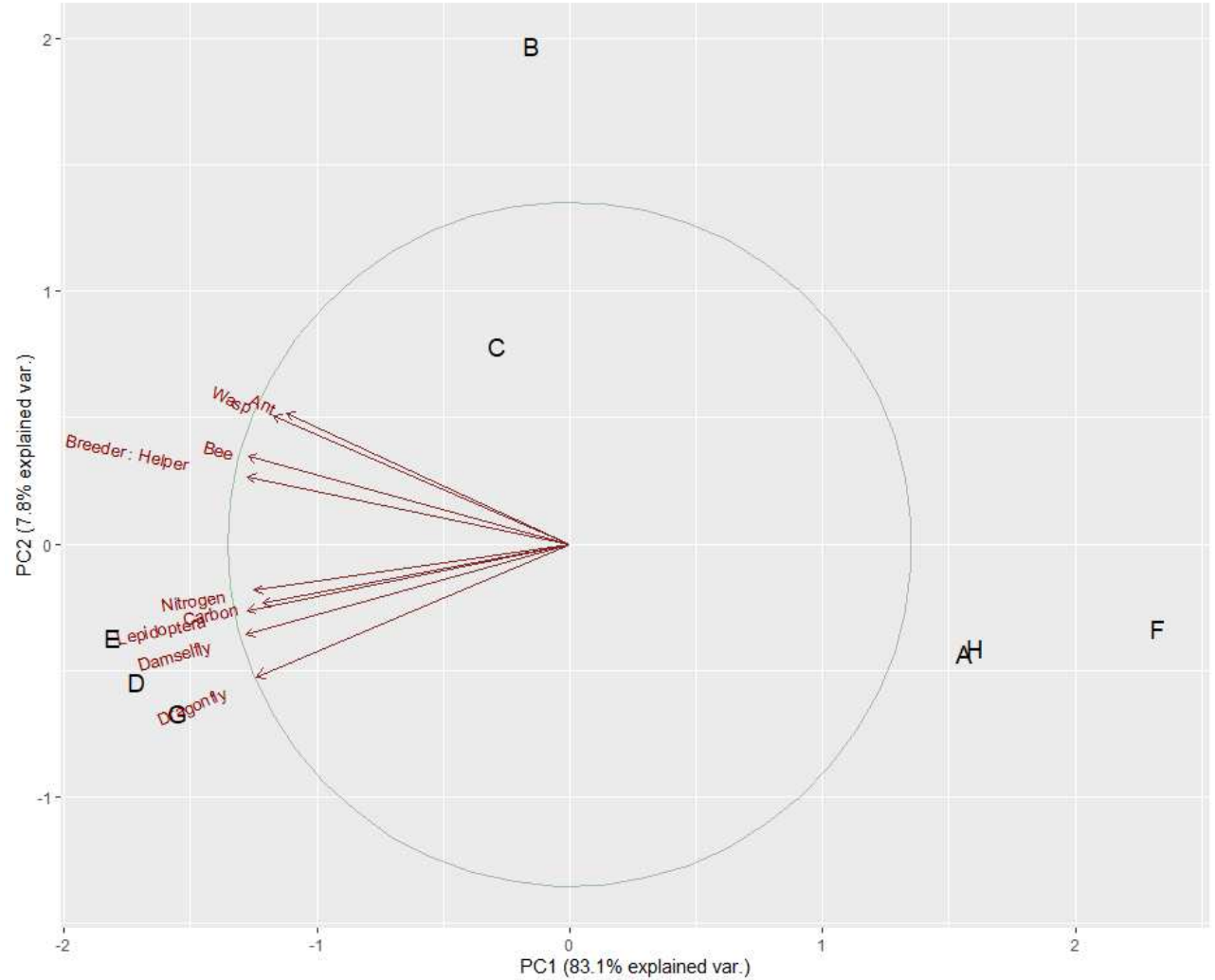

Figure 14: PCA of all correlated factors for reproductive success

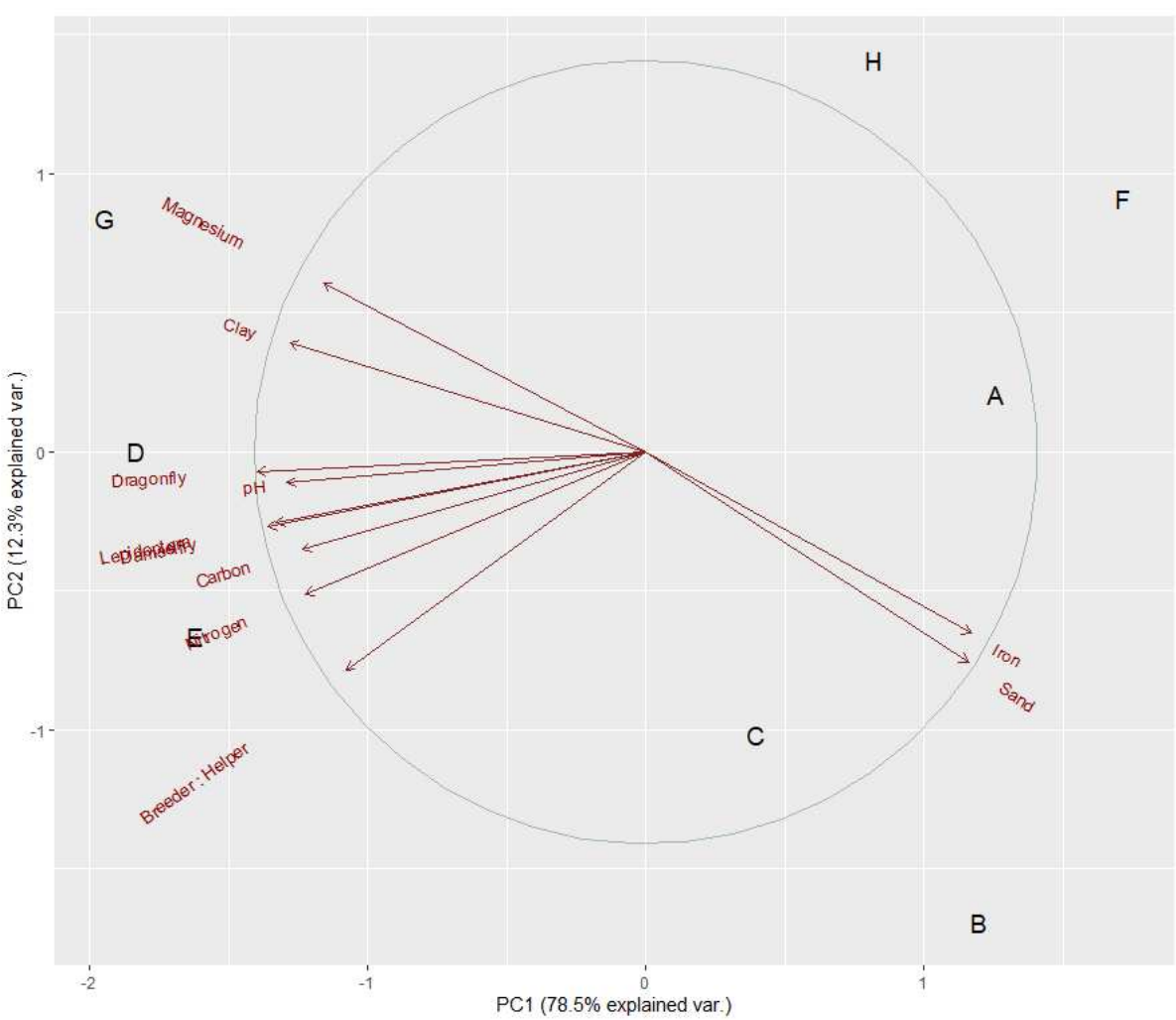




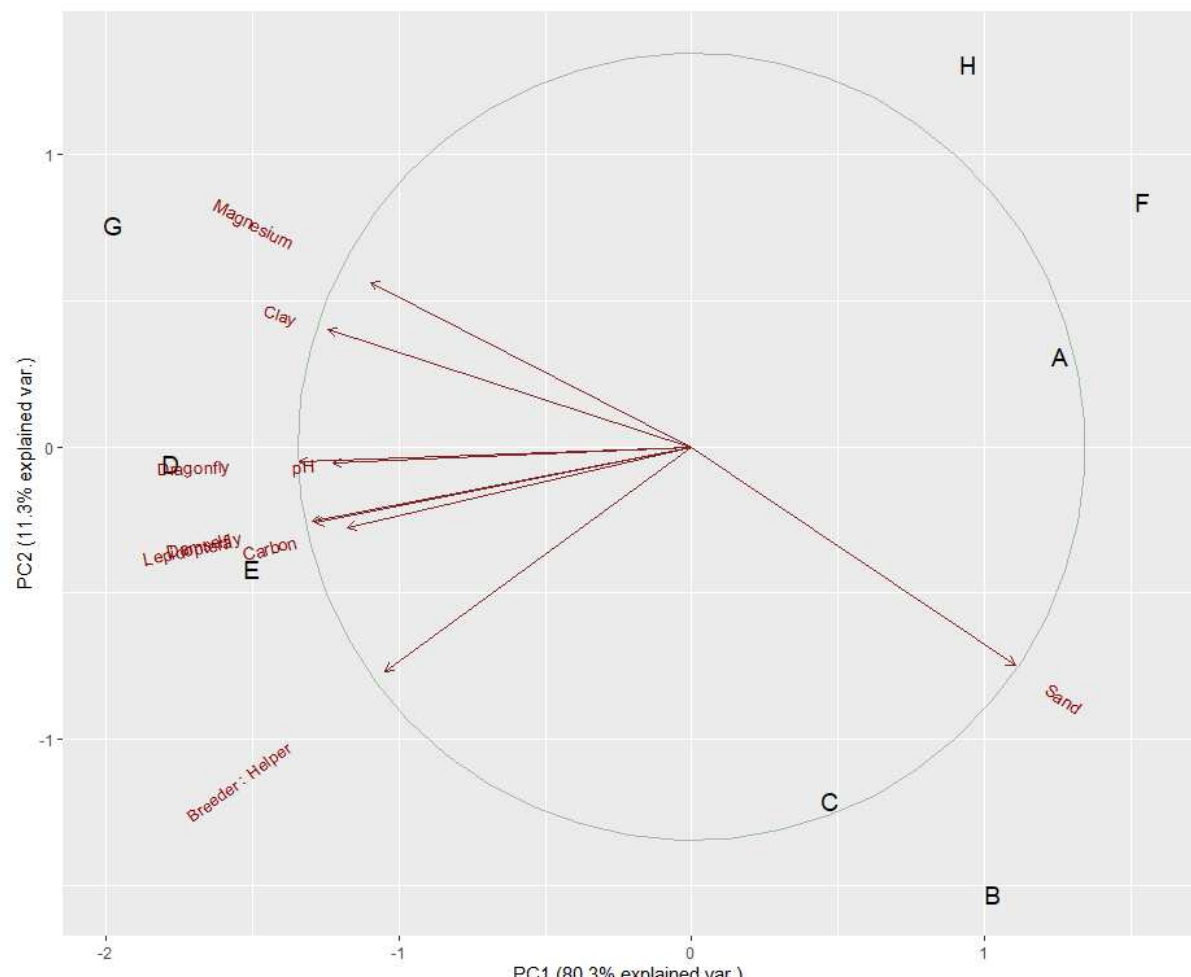

Figure 16: PCA of all correlated factors for mating success

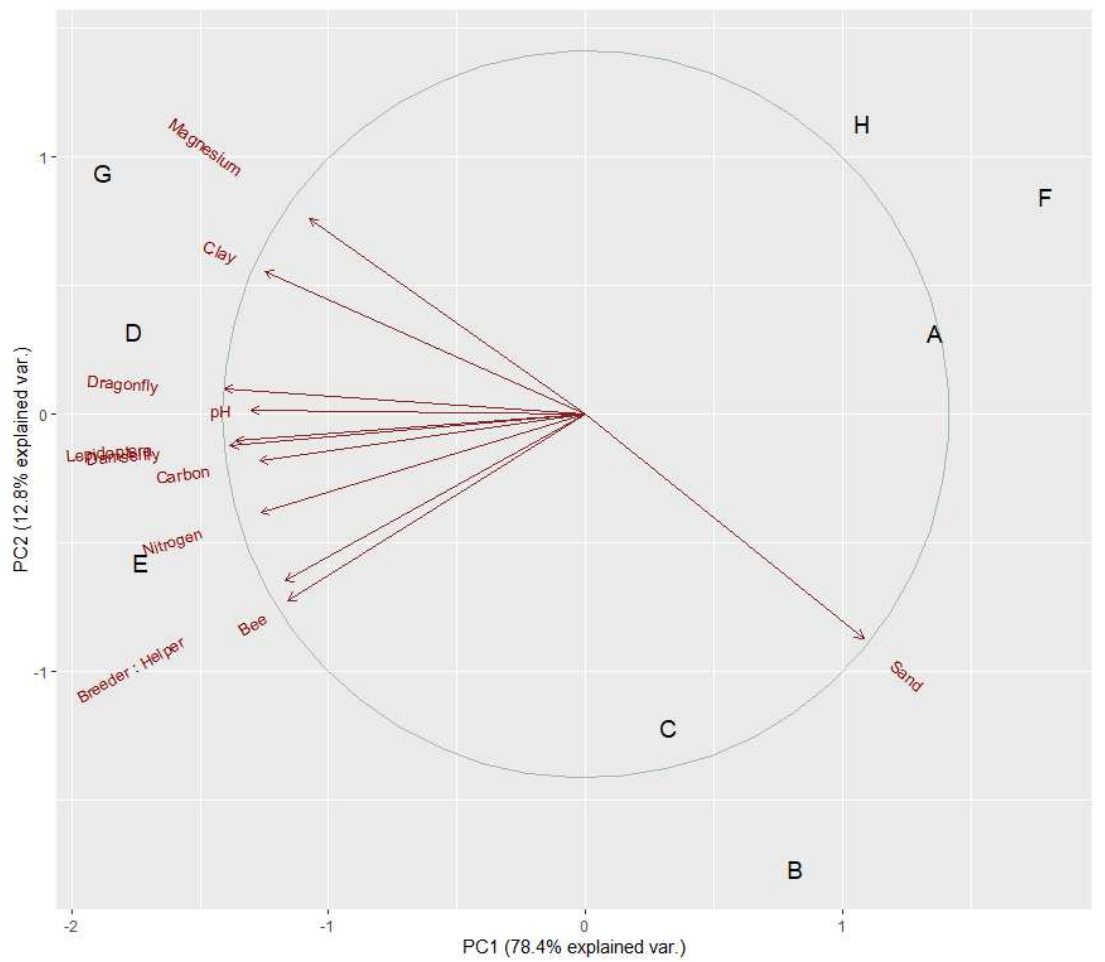

Figure 17: PCA of all correlated factors for hatching success 


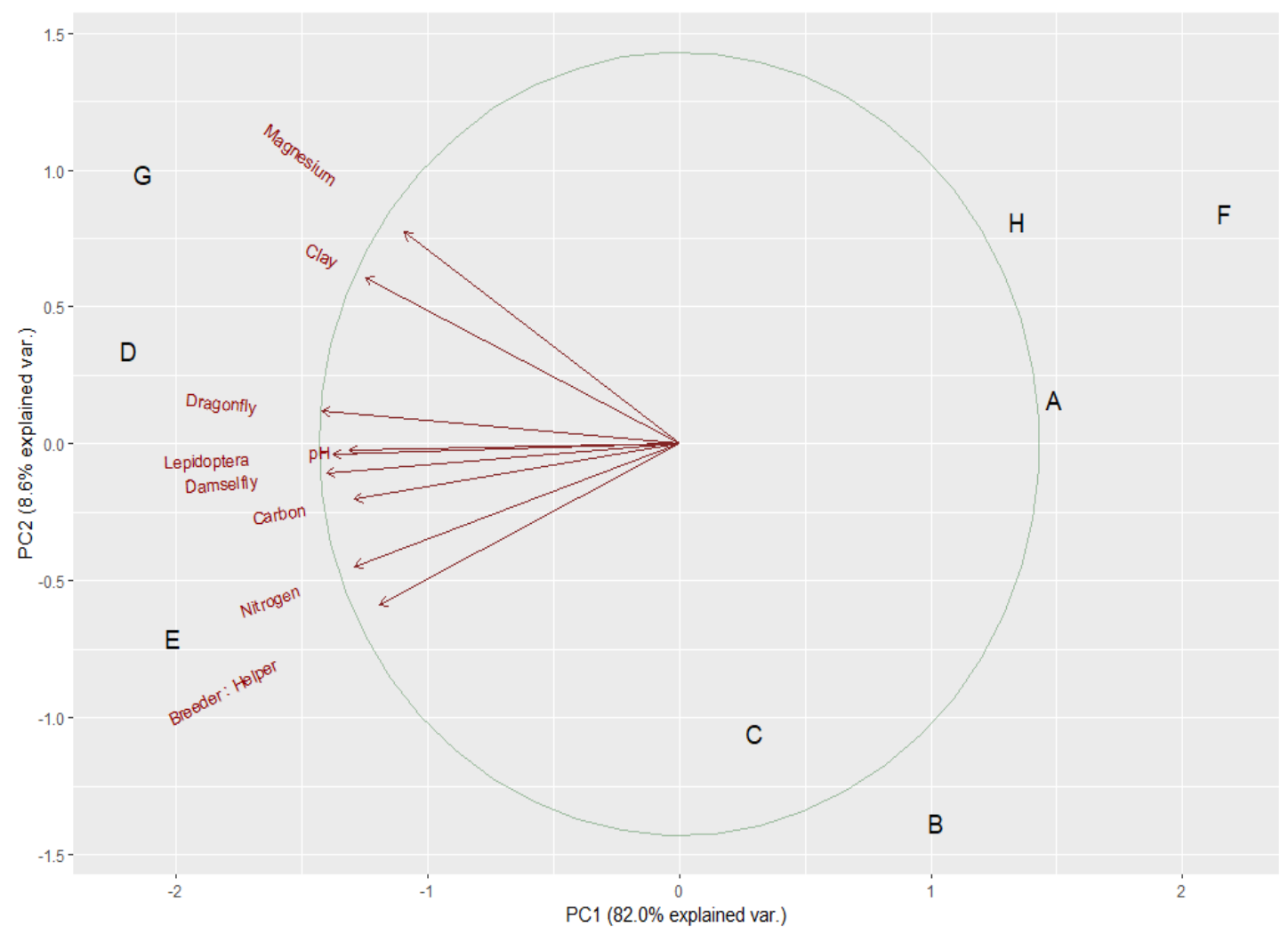

Figure 18: PCA of all correlated factors for fledging

\section{Appendix B}

470 Table 1: Structure of ANNs for best prediction as per gridsearch

\begin{tabular}{|c|c|c|c|c|c|}
\hline $\begin{array}{l}\text { Input variable } \\
\text { set }\end{array}$ & $\begin{array}{l}\text { Number of } \\
\text { input }\end{array}$ & $\begin{array}{l}\text { Output } \\
\text { variables }\end{array}$ & Output type & $\begin{array}{l}\text { Number of } \\
\text { nodes per first } \\
\text { hidden layer }\end{array}$ & $\begin{array}{l}\text { Number of } \\
\text { nodes per } \\
\text { second hidden } \\
\text { layer }\end{array}$ \\
\hline \multirow{10}{*}{$\begin{array}{l}\text { All identified } \\
\text { predictors }\end{array}$} & \multirow[t]{10}{*}{23} & \multirow{2}{*}{$\begin{array}{l}\text { Reproductive } \\
\text { success }\end{array}$} & Non linear & 10 & 2 \\
\hline & & & linear & 14 & 3 \\
\hline & & \multirow[t]{2}{*}{ Nesting success } & Non linear & 3 & 2 \\
\hline & & & linear & 13 & 3 \\
\hline & & \multirow[t]{2}{*}{ Mating success } & Non linear & 5 & 2 \\
\hline & & & linear & 3 & 2 \\
\hline & & \multirow{2}{*}{$\begin{array}{l}\text { Hatching } \\
\text { success }\end{array}$} & Non linear & 5 & 2 \\
\hline & & & linear & 5 & 3 \\
\hline & & \multirow{2}{*}{$\begin{array}{l}\text { Fledging } \\
\text { success }\end{array}$} & Non linear & 10 & 2 \\
\hline & & & linear & 3 & 1 \\
\hline \multirow{10}{*}{$\begin{array}{l}\text { All correlated } \\
\text { predictors }\end{array}$} & \multirow[t]{2}{*}{9} & \multirow{2}{*}{$\begin{array}{l}\text { Reproductive } \\
\text { success }\end{array}$} & Non linear & 6 & 2 \\
\hline & & & linear & 6 & 2 \\
\hline & \multirow[t]{2}{*}{11} & \multirow[t]{2}{*}{ Nesting success } & Non linear & 5 & 2 \\
\hline & & & linear & 6 & 2 \\
\hline & \multirow[t]{2}{*}{9} & \multirow[t]{2}{*}{ Mating success } & Non linear & 6 & 2 \\
\hline & & & linear & 5 & 2 \\
\hline & \multirow[t]{2}{*}{11} & \multirow{2}{*}{$\begin{array}{l}\text { Hatching } \\
\text { success }\end{array}$} & Non linear & 9 & 2 \\
\hline & & & linear & 8 & 2 \\
\hline & \multirow[t]{2}{*}{9} & \multirow{2}{*}{$\begin{array}{l}\text { Fledging } \\
\text { success }\end{array}$} & Non linear & 9 & 2 \\
\hline & & & linear & 5 & 2 \\
\hline
\end{tabular}




\section{Supplementary Files}

This is a list of supplementary files associated with this preprint. Click to download.

- Nestingsuccessmodelsfinal.rmd

- nestingsocio.csv 